Pacific Journal of Mathematics

THE AMBIENT HOMEOMORPHY OF AN INCOMPLETE 


\title{
THE AMBIENT HOMEOMORPHY OF AN INCOMPLETE SUBSPACE OF INFINITE-DIMENSIONAL HILBERT SPACES
}

\author{
JAMES E. WEST
}

\begin{abstract}
The pair $\left(H, H_{f}\right)$ is studied from a topological point of view (where $H$ is an infinite-dimensional Hilbert space and $H_{f}$ is the linear span in $H$ of an orthonormal basis), and a complete characterization is obtained of the images of $H_{f}$ under homeomorphisms of $H$ onto itself. As the characterization is topological and essentially local in nature, it is applicable in the context of Hilbert manifolds and provides a characterization of $\left(H, H_{f}\right)$-manifold pairs $(M, N)$ (with $M$ an $H$-manifold and $N$ an $H_{f}$-manifold lying in $M$ so that each coordinate chart $f$ of $M$ may be taken to be a homeomorphism of pairs $\left.(U, U \cap N) \stackrel{f}{\longrightarrow}\left(f(U), f(U) \cap H_{f}\right)\right)$.

This implies that in the countably infinite Cartesian product of $H$ with itself, the infinite (weak) direct sum of $H_{f}$ with itself is homeomorphic to $H_{f}$ (the two form such a pair), and that if $K$ is a locally finite-dimensional simplicial complex equipped with the barycentric metric (inducing the Euclidean metric on each simplex) and if no vertex-star of $K$ contains more than $\operatorname{dim}(H)$ vertices, then $\left(K \times H, K \times H_{f}\right)$ is an $\left(H, H_{f}\right)$-manifold pair.
\end{abstract}

These results are used in [10] to study $H_{f}$-manifolds much more intensively to obtain results previously available only for $H$-manifolds or in the case that $H_{f}$ is separable, i.e., connected $H_{f}$-manifolds are homeomorphic to open subsets of $H_{f}$, homotopy-equivalent $H_{f}$-manifolds are homeomorphic, and there is an essentially unique completion of an $H_{f}$-manifold into an $H$-manifold, yielding an $\left(H, H_{f}\right)$-pair.

It should be remarked that this characterization has already been achieved for separable Hilbert spaces by R. D. Anderson [1] and by C. Bessaga and A. Pełczynski [5], and that the observations concerning $\left(H, H_{f}\right)$-manifold pairs have been made by T. A. Chapman $[6,7]$ in that case. (Chapman then proceeded to obtain most of the results of [10] in the separable case by methods which seem at the moment to be limited to separability.)

Throughout the discussion, $X$ will denote some complete metric space, and $\mathscr{H}(X)$, the group of all homeomorphisms of $X$ onto itself. The term "isotopy" ("isotopic") will be understood as an abbreviation for "invertible, ambient isotopy", that is, a map $F: X \times[0,1] \rightarrow X$ such that the function $G: X \times[0,1] \rightarrow X \times[0,1]$ defined from $F$ by setting $G(x, t)=(F(x, t), t)$ is a homeomorphism. (When an embedding 
$f$ of a subset of $X$ into $X$ is said to be isotopic to the identity, then, there will exist an extension $g$ of $f$ to an element of $\mathscr{H}(X)$ which is invertibly ambient isotopic to the identity.) If $\mathscr{C}$ is a collection of open sets of $X$, a map $f$ of a subset $Y$ of $X$ into $X$ will be said to be limited by $\mathscr{C}$ if for each point $y$ of $Y$ such that $y \neq f(y)$, there is a member of $\mathscr{C}$ containing both. A homotopy $F: Y \times[0,1] \rightarrow X$ will be said to be limited by $\mathscr{C}$ if for each point $y$ of $Y$ such that $F(\{y\} \times[0,1]) \neq\{y\}$, there is an element of $\mathscr{U}$ containing $F(\{y\} \times[0,1])$. If $\mathscr{S}$ is a collection of subsets of $X$ then $\mathscr{S}^{*}$ will denote their union, and $\mathscr{S}$ will be termed normal whenever there is an open cover $\mathscr{U}$ of $\mathscr{S}^{*}$ by mutually disjoint sets with the

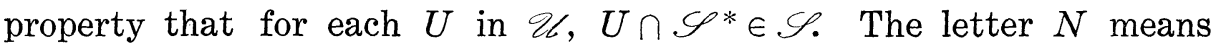
the positive integers. Finally, if $A$ is a subset of $X$ and $\mathscr{S}$ is a collection of subsets of $X$, then st $(A, \mathscr{S})$ denotes the star of $A$ with respect to $\mathscr{S}$, that is, the union of all members of $\mathscr{S}$ meeting $A$, and $\operatorname{st}(\mathscr{S})=\{$ st $(S, \mathscr{S}) \mid S \in \mathscr{S}\}$. Also,

$$
\operatorname{st}^{n}(A, \mathscr{S})=\operatorname{st}\left(\operatorname{st}^{n-1}(A, \mathscr{S}), \mathscr{S}\right),
$$

and $\operatorname{st}^{n}(S)=s t\left(\operatorname{st}^{n-1}(\mathscr{S})\right)$. All refinements used will be understood to be composed of open sets, and $\mathscr{T}$ is a st $^{n}$-refinement of $\mathscr{S}$ provided that $\operatorname{st}^{n}(\mathscr{T})$ refines $\mathscr{S}$.

The first lemma is due to Anderson and Bing [2].

LEMMA 1. Let $\left\{f_{n}\right\}_{n \in N}$ be a sequence of homeomorphisms of the complete metric space $X$ onto itself, and let $\mathscr{U}$ be any open cover of $X$. If $\left\{U_{n}\right\}_{n=0}^{\infty}$ is a collection of open covers of $X$ such that

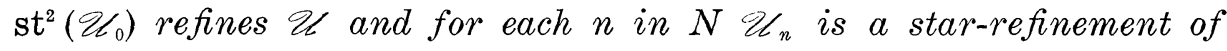
$\mathscr{U}_{n-1}$ of mesh less than $1 / 2^{n}$, then $\left\{f_{n} \circ \ldots \circ f_{1}\right\}_{n \in N}$ converges (uniformly) to a member of $\mathscr{H}(X)$ which is limited by $\mathscr{Q}$ provided that for each $n$ in $N f_{n+1}$ is limited by $\mathscr{U}_{n}$ and mesh

$$
\left(f_{1}^{-1} \circ \cdots \circ f_{n}^{-1}\left(\mathscr{C}_{n}\right)\right)<1 / 2^{n} .
$$

Proof. Anderson and Bing proved that $\left\{f_{n} \circ \cdots \circ f_{1}\right\}_{n \in N}$ converges uniformly to a member $f$ of $\mathscr{C}(X)$. To verify that $f$ is limited by $\mathscr{C}$, it is sufficient to observe that for each $x$ in $X$ and $n$ in $N$, there is a $U(x, n)$ in $\mathscr{C}_{n}$ containing both $f_{n} \circ \cdots \circ f_{1}(x)$ and $f_{n+1} \circ \cdots \circ f_{1}(x)$, and there is also a $U(x, 0)$ in $\mathscr{Q}_{0}$ containing both $x$ and $f_{1}(x)$. If $V(x, n)$ is an element of $\mathscr{Q}_{n-1}$ containing st $\left(U(x, n), \mathscr{C}_{n}\right)$ for each $x$ and $n$, then $x$ and $f_{n+1} \circ \cdots \circ f_{1}(x)$ lie in

$$
\begin{aligned}
\bigcup_{m=0}^{n} U(x, m) & \subset \bigcup_{m=0}^{n-1} U(x, m) \cup V(x, n) \\
& \subset \bigcup_{m=0}^{n-2} U(x, m) \cup V(x, n-1) \subset \cdots \subset U(x, 0) \cup V(x, 1) \\
& \subset \operatorname{st}\left(U(x, 0), \mathscr{Q}_{0}\right),
\end{aligned}
$$


so $x$ and $f(x)$ must lie in the closure of st $\left(U(x, 0), \mathscr{C}_{0}\right)$, which is contained in $\operatorname{st}^{2}\left(U(x, 0), \mathscr{U}_{0}\right)$, which lies in some member of $\mathscr{U}$.

LEMMA 2. If $\mathscr{Q}$ is a collection of pairwise disjoint open subsets of $X$, then there is an open cover $\mathscr{Y}$ of $\mathscr{U}^{*}$, refining $\mathscr{U}$, with the property that if for each $U \in \mathscr{C} f_{U}$ is a homeomorphism of $U$ onto itself which is limited by $\mathscr{Y}$, then the function $f$ defined by $f(x)=$ $f_{U}(x)$, if $x \in U$, and $f(x)=x$, if $x \notin \mathscr{Q}^{*}$, is a homeomorphism of $X$ onto itself.

Proof. Let $\mathscr{Y}=\{V(x)=\{y \in X \mid d(y, x)<d(z, x) / 2$ for each $z$ in $X \backslash U\} \mid x \in U \in \mathscr{Q}\}$, where $d(\cdot, \cdot)$ is the metric for $X$. Then for any points $z$ of $X \backslash U^{*}$, and $y$ of $X, d(z, f(y)) \leqq 3 d(z, y)$, which establishes continuity. As $f$ must be one-to-one and onto, and the same argument establishes the continuity of $f^{-1}, f$ is a homeomorphism.

Let $\mathscr{K}$ be an hereditary collection of closed subsets of $X$ which is invariant under the action of $\mathscr{C}(X)$, that is, each closed subset of a member of $\mathscr{K}$ is in $\mathscr{K}$ and $f(K) \in \mathscr{K}$ if $K \in \mathscr{K}$ and $f \in \mathscr{K}(X)$. A set $A$ in $X$ will be termed $\mathscr{K}$-absorptive if for each open cover $\mathscr{U}$ of a member $K$ of $\mathscr{K}$ and each member $K^{\prime}$ of $\mathscr{K}$ contained in $K \cap A$, there is a homeomorphism $f$ in $\mathscr{C}(X)$ which is limited by $\mathscr{C}$, is the identity on $K^{\prime}$, and carries $K$ into $A$. If $f$ may always be chosen so that there is an isotopy from it to the identity which is limited by $\mathscr{Q}$, then $A$ will be called strongly $\mathscr{K}$-absorptive.

Lemma 3. If $A$ is $\mathscr{K}$-absorptive (strongly $\mathscr{K}$-absorptive), $L$ is an open subset of a member of $\mathscr{K}$, and $U$ is an open cover of $L$ in $X$, then there is a member $f$ of $\mathscr{L}(X)$ carrying $L$ into $A$ which is limited by $\mathscr{U}$ (is isotopic to the identity by an isotopy limited by $\mathscr{C}$ ).

Proof. As $\mathscr{C}^{*}$ is an open subset of the complete metric space $X$, it may be given an equivalent metric under which it is itself complete, so Lemma 1 holds under the new metric. Let $\left\{V_{n}\right\}_{n \in N}$ be a sequence of open sets in $X$ such that each contains its successor and $\bigcap_{n \in N} V_{n}=X \backslash U^{*}$, and let $\mathscr{W}$ be a refinement of $\mathscr{C}$ which covers $\mathscr{U}^{*}$ and has the property that any member of $\mathscr{H}\left(\mathscr{C}^{*}\right)$ which is limited by $\mathscr{W}$ extends to an element of $\mathscr{K}(X)$ which is also limited by $\mathscr{W}$. If $\mathscr{K}^{\prime}$ is the collection of all members of $\mathscr{K}^{\prime}$ which lie in $\mathscr{U}^{*}$, then from the definition of (strong) $\mathscr{K}$-absorptivity it is immediate that as a subset of $\mathscr{C}^{*}, A \cap U^{*}$ is (strongly) $\mathscr{K}^{\prime}$ absorptive. Using Lemma 1 and the fact that $L \backslash V_{n+1}$ contains $L \backslash V_{n}$ for all $n$ in $N$ and that both are in $\mathscr{K}^{\prime}$, select a sequence $\left\{f_{n}\right\}_{n \in N}$ of members of $\mathscr{H}\left(\mathscr{U}^{*}\right)$ with $\left\{f_{n} \circ \cdots \circ f_{1}\right\}_{n \in N}$ converging to a member of $\mathscr{C}\left(\mathscr{C}^{*}\right)$ which is limited by $\mathscr{W}$ and such that for each $n, f_{n}$ 
carries $f_{n-1} \circ \cdots \circ f_{1}\left(L \backslash V_{n}\right)$ into $A \cap \mathscr{Q}^{*}$ and is the identity on

$$
f_{n-1} \circ \cdots \circ f_{1}\left(L \backslash V_{n-1}\right) \text {. }
$$

This may be done because each of the functions $f_{n} \circ \cdots \circ f_{1}$ may be kept limited by $\mathscr{W}$, which ensures that they permute the elements of $\mathscr{K}^{\prime}$. Extending the limit homeomorphism to all of $X$ so that it is the identity off $\mathscr{U}^{*}$ produces the desired member of $\mathscr{H}(X)$. (In the case that an isotopy is desired, and that $A$ is strongly $\mathscr{K}$-absorptive, consider the cover $\mathscr{W}^{\prime}=\{W \times[0,1] \mid W \in \mathscr{W}\}$ of $\mathscr{Q}^{*} \times[0,1]$ and construct a level-preserving homeomorphism of $\mathscr{C}^{*}$ which is limited by $\mathscr{\mathscr { W }}^{\prime}$, is the identity on $\mathscr{C}^{*} \times\{0\}$, and carried $L \times\{1\}$ into $A \times\{1\}$. The associated isotopy extends to $X$.)

A collection $\mathscr{A}$ of members of $K$ will be called a $\mathscr{K}$-complex if it may be expressed as a countable union $\bigcup_{n=0}^{\infty} \mathscr{A}_{n}$ of subsets of itself such that $\mathscr{A}^{n}=\mathrm{U}_{m=0}^{n} \mathscr{A}_{m}^{*}$ is closed for each $n$ and $\mathscr{A}[n]=$ $\left\{A\left|\mathscr{A}^{n-1}\right| A \in \mathscr{A}_{n}\right\}$ is normal for all $n$. (Here, $\mathscr{A}^{-1}=\varnothing$.) The set $\mathscr{A}^{*}$ will be said to admit the structure of a $\mathscr{K}$-complex. If $\mathscr{A}^{*}$ is (strongly) $\mathscr{K}$-absorptive, then it will be referred to as a (strong) $\mathscr{K}$-absorption base.

THeorem 1. If $\mathscr{U}$ is an open cover of $X$ and $A^{*}$ and $B^{*}$ are two (strong) $\mathscr{K}$-absorption bases in $X$, there is a homeomorphism $f$ of $X$ onto itself (an isotopy $F$ of $X$ ), limited by $\mathscr{U}$, such that $f\left(A^{*}\right)=B^{*}\left(F\left(A^{*} \times\{1\}\right)=B^{*}\right)$.

Proof. Let $\mathscr{A}=\bigcup_{n=0}^{\infty} \mathscr{A}_{n}$ and $\mathscr{B}=\bigcup_{n=0}^{\infty} \mathscr{B}_{n}$ be $\mathscr{K}$-complex structures for $A^{*}$ and $B^{*}$ respectively. As the construction of an isotopy in the strong case may be handled from the construction of a homeomorphism in the other case as was done in the previous proof, only the latter construction will be made here. It is quite simple. Since $\mathscr{K}$ is invariant under the action of $\mathscr{H}(X)$, so is the collection of (strong) $\mathscr{K}$-absorption bases. A sequence $f_{1}, g_{1}, f_{2}, g_{2}, \ldots$ of members of $\mathscr{H}(X)$ is to be chosen with $\left\{g_{n}^{-1} \circ f_{n} \circ \cdots \circ g_{1}^{-1} \circ f_{1}\right\}_{n \in N}$ converging to an element $f$ of $\mathscr{H}(X)$ which is limited by $\mathscr{U}$. Furthermore, $f_{n}\left(g_{n-1}^{-1} \circ \ldots \circ f\left(\mathscr{A}^{n}\right)\right)$ is to be a subset of $\mathscr{B}^{*}, g_{n}\left(\mathscr{B}^{n}\right)$ is to be a subset of $f_{n} \circ g_{n-1}^{-1} \circ \cdots \circ g_{1}^{-1} \circ f_{1}\left(\mathscr{A}^{*}\right), f_{n}$ is to be the identity on $g_{n-1}^{-1} \circ f_{n-1} \circ \cdots \circ g_{1}^{-1} \circ f_{1}\left(\mathscr{A}^{n-1}\right) \cup \mathscr{B}^{n-1}$, and $g_{n}$ is to be the identity on $f_{n} \circ g_{n-1}^{-1} \circ \cdots \circ g_{1}^{-1} \circ f_{1}\left(\mathscr{A}^{n}\right) \cup \mathscr{B}^{n-1}$. Then the limit homeomorphism $f$ is limited by $\mathscr{C}$ and $f\left(\mathscr{A}^{*}\right)=\mathscr{B}^{*}$. The selection of these homeomorphisms may be made inductively so as to satisfy the convergence criterion of Lemma 1 because for each $n, \mathscr{A}[n]$ and $\mathscr{B}[n]$ are normal and $\mathscr{A}^{n-1}$ and $\mathscr{B}^{n-1}$ are closed, so Lemmas 2 and 3 may be applied and the homeomorphisms constructed piecemeal on collections of pairwise disjoint open sets in $X$. 
THEOREM 2. If $U$ is an open subset of $X, A^{*}$ is a (strong) $\mathscr{K}$-absorption base for $X$, and $\mathscr{K}^{\prime}$ is the set of all members of $\mathscr{K}$ contained in $U$, then $A^{*} \cap U$ is a (strong) $\mathscr{K}^{\prime}$-absorption base for $U$.

Proof. It has already been remarked that $A^{*} \cap U$ is (strongly) $\mathscr{K}^{\prime \prime}$-absorptive, so all that is necessary is to demonstrate that it admits the structure of a $\mathscr{K}^{\prime}$-complex. If $A^{*} \cap U=\varnothing$, then $\mathscr{K}^{\prime}=$ $\{\varnothing\}$, and $A^{*} \cap U$ is a strong $\mathscr{K}^{\prime}$-absorption base for $U$. Otherwise, let $\left\{V_{n}\right\}_{n \in N}$ be a collection of open sets with $X \backslash U \subset V_{n+1} \subset \bar{V}_{n+1} \subset V_{n}$ for each $n$, and with $\bigcap_{n \in N} V_{n}=X \backslash U$. Now, let

$$
\mathscr{B}_{2 n}=\bigcup_{m=0}^{n}\left\{A \backslash V_{2(n-m+1)} \mid A \in \mathscr{A}_{m}\right\}
$$

and $\mathscr{B}_{2 n+1}=\bigcup_{m=0}^{n+1}\left\{A \backslash V_{2(n-m+1)+1} \mid A \in \mathscr{A}_{m}\right\}$. If $\bigcup_{n=0}^{\infty} \mathscr{B}_{n}$ is denoted by $\mathscr{B}$, it is apparent that $\mathscr{B}^{n}$ is closed for each $n$. To see that $\mathscr{B}[n]$ is normal for each $n$, let $\left\{\mathscr{U}_{n}\right\}_{n \in N}$ be a collection of sets of mutually disjoint open sets of $X$ with the property that $\mathscr{U}_{n}{ }^{*}$ contains $\mathscr{A}[n]^{*}$ and that for each $U$ in $\mathscr{U}_{n}, U \cap \mathscr{A}[n]^{*} \in \mathscr{A}[n]$. Then define $\mathscr{W}_{2 n}=$ $\bigcup_{m=0}^{n}\left\{U \cap V_{2(n-m)+1}\left|\bar{V}_{2(n-m+1)+1}\right| U \in \mathscr{Q}_{m}\right\}$ and

$$
\left.\mathscr{W}_{2 n+1}=\bigcup_{m=0}^{n+1}\left\{U \cap V_{2(n-m+1}\right) \backslash \bar{V}_{2(n-m+2)} \mid U \in \mathscr{U}_{m}\right\},
$$

for each $n=0,1, \cdots$ The collections $\mathscr{W}_{n}$ are composed of pairwise disjoint open sets separating members of $\mathscr{B}[n]$, so $\mathscr{B}$ is a $\mathscr{K}^{\prime}$ complex. Since $\mathscr{B}^{*}=\mathscr{A}^{*} \cap U$, the proof is complete.

If $\left\{Y_{n}\right\}_{n \in N}$ is a collection of spaces, then $\prod_{n \in N} Y_{n}$ will denote their Cartesian product. If, for each $n, y_{n} \in Y_{n}$, then $\prod_{n \in N}\left(Y_{n}, y_{n}\right)$ will denote that subset of $\prod_{n \in N} Y_{n}$ composed of those points with $n$-th coordinate differing from $y_{n}$ for at most finitely many $n$. Also, let $\mathscr{E}$ be a class of spaces which is closed under the operations of taking closed subsets and of taking finite products, and for each space $Y$, let $\mathscr{C}(Y)$ denote the collection of images of members of $\mathscr{C}$ under closed embeddings in $Y$.

THEOREM 3. If $\left\{X_{n}\right\}_{n \in N}$ is a sequence of complete metric spaces and if, for each $n, \mathscr{A}(n)$ is a $\mathscr{C}\left(X_{n}\right)$-complex, and $x_{n}$ is a point of $\mathscr{A}(n)^{*}$, then $\prod_{n \in N}\left(\mathscr{A}(n)^{*}, x_{n}\right)$ admits the structure of a $\mathscr{C}\left(\prod_{n \in N} X_{n}\right)$ complex.

Proof. For each finite subset $S$ of $N$, let $f$ denote the natural injection of $\prod_{n \in S} X_{n}$ into $\prod_{n \in N}\left(X_{n}, x_{n}\right)$. Now, for each ordered $n$-tuple $\left(m_{1}, \cdots, m_{n}\right)$ of nonnegative integers, each of which is no greater than $n$, let $\mathscr{B}\left(n ; m_{1}, \cdots, m_{n}\right)=\left\{f\left(\prod_{i=1}^{n} A_{i}\right) \mid A_{i} \in \mathscr{A}(i)_{m_{i}}\right\}$. Order 
the set of all these collections in such a manner that

$$
\mathscr{B}\left(n ; m_{1}, \cdots, m_{n}\right) \geqq \mathscr{B}\left(n^{\prime} ; m_{1}^{\prime}, \cdots, m_{n^{\prime}}^{\prime}\right)
$$

if $n \geqq n^{\prime}$ or if $n=n^{\prime}$ and $m_{j} \geqq m_{j}^{\prime}$ for all $j$. The order selected will be isomorphic to the nonnegative integers, so index the $\mathscr{B}$ 's by them in a manner consistent with the above requirements. Let $\mathscr{B}=\bigcup_{n=0}^{\infty} \mathscr{B}_{n}$. For each $n, \mathscr{B}_{n}{ }^{*}$ is closed, so $\mathscr{B}^{n}$ is, also. Thus, in order to check that $\mathscr{B}$ is a $\mathscr{C}\left(\prod_{n \in N} X_{n}\right)$-complex, it is only necessary to verify that $\mathscr{B}[i]$ is normal for each $i$. However, for $n$ and $\left(m_{1}, \cdots, m_{n}\right)$ such that $\mathscr{B}_{i}=\mathscr{B}\left(n ; m_{1}, \cdots, m_{n}\right)$, and for $B$ in $\mathscr{B}_{i}, B \backslash \mathscr{B}^{i-1} \subset B \backslash f\left(\prod_{j=1}^{n} \mathscr{A}(j)^{m_{j-1}}\right)$, so if for each $n$ in $N$ and each nonnegative integer $m, \mathscr{C}_{m}^{n}$ is an open cover of $\mathscr{A}(n)[m]^{*}$ in $X_{n}$ by pairwise disjoint open sets $U$ with the property that

$$
U \cap \mathscr{A}(n)[m]^{*} \in \mathscr{A}(n)[m],
$$

then $\mathscr{V}_{i}=\left\{\prod_{j=1}^{n} U_{j} \times \prod_{j=n+1}^{\infty} X_{j} \mid U_{j} \in \mathscr{U}_{m_{j}}^{j}\right.$ for $\left.j=1, \cdots, n\right\}$ is a cover of $\mathscr{B}[i]$ by mutually disjoint open sets of $\Pi_{n \in N} X_{n}$ with the property that the intersection of each with $\mathscr{B}[i]^{*}$ is a member of $\mathscr{B}[i]$. Thus, each $\mathscr{B}[i]$ is normal and $\mathscr{B}$ is a $\mathscr{C}\left(\prod_{n \in N} X_{n}\right)$-complex. As it is immediate that $\mathscr{B}^{*}=\prod_{n \in N}\left(\mathscr{A}(n)^{*}, x_{n}\right)$, the theorem has been proved.

REMARK. It was tacitly assumed above that there were infinitely many $X_{n}^{\prime} s$. Of course, the same proof works for a finite collection.

Corollary 1. If, in the above, $\prod_{n \in N}\left(\mathscr{A}(n)^{*}, x_{n}\right)$ is (strongly) $\mathscr{C}\left(\prod_{n \in N} X_{n}\right)$-absorptive, then it is a (strong) $\mathscr{C}\left(\prod_{n \in N} X_{n}\right)$-absorption base.

REMARK. It is clear from the definitions that if $X$ and $Y$ are homeomorphic, then any homeomorphism between them carries the $\mathscr{C}(X)$-complexes to the $\mathscr{C}(Y)$-complexes and the (strong) $\mathscr{C}(X)$ absorption bases to the (strong) $\mathscr{C}(Y)$-absorption bases.

From now on, $\mathscr{C}$ will denote the class of all finite-dimensional compact metric spaces. The next lemma is an extension of Proposition 4.5 of [5] to the nonseparable case and to isotopies. It consists of combining Theorem 4.2 of [3] with the Bartle-Graves Theorem.

Lemma 4. If $X$ is an infinite-dimensional Fréchet space and $K$ is a compact subset of $X$, then for each open cover $\mathscr{Q}$ of $K$ there is a second, $\mathscr{Y}$, such that any embedding of $K$ in $X$ which is limited by $\mathscr{Y}$ is (invertibly ambient) isotopic to the identity by an isotopy which is limited by $\mathscr{U}$. 
Proof. For a real number (positive) $r$ and a point $x$ in a metric space, $N(x, r)$ will denote the open ball centered at $x$ with radius $r$.

Let $\lambda$ be a Lebesgue number of $\mathscr{U}$ with respect to $K$, let $\mathscr{V}_{1}=\left\{N\left(x, \lambda / 3^{6}\right) \mid x \in K\right\}$, and, inductively, for $n>1$, let

$$
\mathscr{V}_{n}=\left\{N\left(x, \lambda / 3^{n+5}\right) \mid x \in \mathscr{\mathscr { V }}_{n-1}^{*}\right\} \text {. }
$$

Now, let $\mathscr{V}=\bigcup_{n \in N} \mathscr{V}_{n}$. If $f$ embeds $K$ in $X$ and is limited by $\mathscr{V}$, let $Y$ be the closed linear span in $X$ of the image of $F: K \times[0,1] \rightarrow X$ defined by $F(x, t)=(1-t) x+t f(x)$. Let $p_{Y}: X \rightarrow X / Y$ be the canonical projection, and let $q_{Y}: X / Y \rightarrow X$ be a right inverse for $p_{Y}$ sending 0 to 0 . (This is by the Bartle-Graves Theorem. For a proof see [11].) Now, the function $h_{f}: X / Y \times Y \rightarrow X$ defined by $h_{f}=q_{Y} p_{1}+p_{2}$ is a homeomorphism, where $p_{1}$ and $p_{2}$ denote the projections onto the first and second factors, respectively.

From the definition of $\mathscr{C}$, it follows that for each element $V$ of st $^{4}(\mathscr{C}), V+N(0, \lambda / 3)$ is contained in some member of $\mathscr{C}$, where here "+" denotes the set of all sums of pairs of elements, one from the first set and one from the second. Letting $W$ be a neighborhood of the origin in $X / Y$ which $q_{Y}$ carries into $N(0, \lambda / 3)$, one sees that $h_{f}\left(W \times\left(\mathscr{V}^{*} \cap Y\right)\right)$ lies in $\mathscr{C}^{*}$ and, indeed, that $\left\{h_{f}(W \times V) \mid V \in \mathrm{st}^{4}(\mathscr{V} \mid Y)\right\}$ refines $\mathscr{U} . \quad$ (Here, $\mathscr{V} \mid Y=\{V \cap Y \mid V \in \mathscr{V}\}$.

Select a map $g: X / Y \rightarrow[0,1]$ such that $g^{-1}(0) \supset(X / Y) \backslash W$ and $0 \in g^{-1}(1)$. Since $Y$ is separable and $\mathscr{C}^{*} \cap Y$ is open in $Y$, [3] yields an isotopy $G:\left(\mathscr{V}^{*} \cap Y\right) \times[0,1] \rightarrow \mathscr{C}^{*} \cap Y$ from the identity homeomorphism at $t=0$ to an extension to $\mathscr{F}^{*} \cap Y$ of $f$ at $t=1$ which is limited by $\operatorname{st}^{4}(\mathscr{V} \mid Y)$. Then $H: X \times[0,1] \rightarrow X$ given by

$$
H(x, t)=\left\{\begin{array}{ll}
h_{f}\left(p_{Y}(x), G\left(p_{2} \circ h_{f}^{-1}(x), t \cdot g \circ p_{Y}(x)\right)\right), & \text { if } x \in h_{f}\left(W \times\left(\mathscr{V}^{*} \cap Y\right)\right) \\
x & , \text { if } x \notin h_{f}(W \times(\mathscr{Y} * \cap Y))
\end{array}\right\}
$$

is the desired isotopy.

Let $H$ be an infinite-dimensional (real) Hilbert space, let $E$ be a complete, orthonormal basis for $H$, and denote by $H_{f}$ the collection of all (finite) linear combinations of members of $E$.

THEOREM 4. $H_{f}$ is a strong $\mathscr{C}(H)$-absorption base.

Proof. Two things must be shown, namely, that $H_{f}$ admits the structure of a $\mathscr{C}(H)$-complex and that it is strongly $\mathscr{C}(H)$-adsorptive. To see the first, let $\mathscr{A}_{0}$ be the set of all integral linear combinations of members of $E$. For $n>0$, let

$$
\mathscr{Q}_{n}=\left\{Q_{n}=\left\{\sum_{m=1}^{n} t_{m} e_{m} \mid t_{m} \in[0,1], m=1, \cdots, n\right\} \mid e_{1}, \cdots, e_{n}\right.
$$

are $n$ distinct elements of $E\}$, 
and let $\mathscr{A}_{n}=\left\{A=Q_{n}+x \mid Q_{n} \in \mathscr{Q}_{n}, x \in \mathscr{A}_{0}\right\}$. It is readily seen that $\mathscr{A}=\mathrm{U}_{n=0}^{\infty} \mathscr{A}_{n}$ is a $\mathscr{C}(H)$-complex with $\mathscr{A}^{*}=H_{f}$.

By Lemma 4, in order to demonstrate that $H_{f}$ is strongly $\mathscr{C}(H)$ absorptive one must only show that for each member $K$ of $\mathscr{C}(H)$, each open cover $\mathscr{C}$ of $K$, and for each closed subset $K^{\prime}$ of $K \cap H_{f}$, there is an embedding $f$ of $K$ in $H_{f}$, limited by $\mathscr{U}$, which is the identity on $K^{\prime}$. Since $K$ is compact, there exists a Lebesgue number $\lambda$ for $\mathscr{U}$ with respect to $K$, so one must only find an embedding $f$ of $K$ in $H_{f}$ which moves no point as much as $\lambda$ and is the identity on $K^{\prime}$. However, the total boundedness of $K$ and the denseness in $H$ of $H_{f}$ lead to the existence of a sequence $\left\{e_{i}\right\}_{i \in N}$ in $E$ and a sequence $\{n(i)\}_{i \in N}$ in $N$ such that if $p_{i}$ is the orthogonal projection of $H$ onto the span of $\left\{e_{j}\right\}_{j=n(i-1)+1}^{n(i)}$, then $\left\|\sum_{i=1}^{m} p_{i}(x)-x\right\|<2^{-m-2} \lambda$ for each $m \in N$ and $x \in K$. Also, since $K$ is finite-dimensional, for each set $S$ of $2 \operatorname{dim}(K)+2$ distinct elements of $E$, there is an embedding of $K$ in the unit sphere (=elements of norm one) of the subspace spanned by $S$. Assume that for each $i, n(i)-n(i-1) \geqq 2 \operatorname{dim}(K)+2$, and let $f_{i}$ be an embedding of $K$ in the unit sphere of the span of $\left\{e_{j}\right\}_{j=n(i-1)+1}^{n(i)}$. Now, let g map $K$ into $[0,1]$ such that $K^{\prime}=g^{-1}(0)$, and for each $i$ let $h_{i} \operatorname{map}[0,1]$ into $[0,1]$ such that $h_{1}^{-1}(0)=[0,1 / n(3)]$ and $h_{1}^{-1}(1)=[1 / n(2), 1]$ and for $i>1$,

$$
h_{i}^{-1}(0)=[1 / n(i-1), 1] \cup[0,1 / n(i+2)]
$$

and $h_{i}^{-1}(1)=[1 / n(i+1), 1 / n(i)]$. Finally, set

$$
f(x)=\sum_{i \in N}\left(\max _{j \geqq i}\left\{h_{j} \circ g(x)\right\}\right) p_{i}(x)+\sum_{i \in N} 2^{-i-1} \lambda \cdot h_{i} \circ g(x) f_{i+3}(x) .
$$

This is the desired embedding.

COROLlary 2. If $\mathscr{C}$ is any collection of open sets of $H$ and $Y$ is any $\mathscr{C}\left(\mathscr{C}^{*}\right)$-absorption base in $\mathscr{U}^{*}$, then there is an ambient, invertible isotopy of $H$ onto itself which is limited by $\mathscr{C}$, is the identity at $t=0$, and at $t=1$ is a homeomorphism $h_{1}$ such that $h_{1}(Y)=\mathscr{U}^{*} \cap H_{f}$.

Proof. Lemma 4 shows the equivalence of the concepts of $\mathscr{C}\left(\mathscr{C}^{*}\right)$ absorption base and strong $\mathscr{C}\left(\mathscr{U}^{*}\right)$-absorption base, Theorem $4 \mathrm{com}$ bined with Theorem 2 gives that $\mathscr{U}^{*} \cap H_{f}$ is also a strong $\mathscr{C}\left(\mathscr{U}^{*}\right)$ absorption base, and Theorem 1 supplies the isotopy on $\mathscr{U}^{*}$ limited by an open cover given by Lemma 2 which refines $\mathscr{U}$ and has the property that any isotopy limited by it may be extended trivially to one on $H$. 
CoRollary 3. Let $\left\{H_{n}\right\}_{n \in N}$ be an indexed, countably infinite collection of copies of $H$, and let $Y$ be the subspace of $\Pi_{n \in N} H_{n}$ consisting of all points with at most finitely many nonzero coordinates, each of which lies in the appropriate copy of $H_{f}$. Then $Y$ is homeomorphic to $H_{f}$.

Proof. It is easy to modify the proof of Theorem 4 to show that $Y$ is $\mathscr{C}\left(\Pi_{n \in N} H_{n}\right)$-absorptive. If the copy of $H_{f}$ in $H_{n}$ is denoted by $\left(H_{f}\right)_{n}$, then $Y=\prod_{n \in N}\left(\left(H_{f}\right)_{n}, 0\right)$, so Corollary 1 applies to show that $Y$ is a $\mathscr{C}\left(\prod_{n \in N} H_{n}\right)$-absorption base. However, $\Pi_{n \in N} H_{n}$ is homeomorphic to $H$ by a theorem of Bessaga and Pełczynski [4], so by the remark following Corollary $1, Y$ may be embedded in $H$ as a $\mathscr{C}(H)$-absorption base. Corollary 2 now applies to finish the proof.

The above result is crucial to [10]. The next two results identify some simplicial complexes whose products with $H_{f}$ are $H_{f}$-manifolds.

THeOREM 5. If $K$ is a metric simplicial complex and $K \times H$ is an $H$-manifold, then $K \times H_{f}$ is an $H_{f}$-manifold.

Proof. By Theorem 3 (the remark after Theorem 3), $K \times H_{f}$ is a $\mathscr{C}(K \times H)$-complex, since $K$ is by definition a $\mathscr{C}(K)$-complex. The strategy of the proof is to show that $K \times H_{f}$ is a $\mathscr{C}(K \times H)$-absorption base, to embed $K \times H$ component-wise in $H$ as open subsets (using a theorem of Henderson [8]) and then to use Corollary 2 to find a homeomorphism of the open subsets in question onto themselves throwing the images of $K \times H_{f}$ onto $H_{f} \cap$ (the open subsets). Thus, all that is necessary is to establish the $\mathscr{C}(K \times H)$-absorptivity of $K \times H_{f}$. In fact, since for each vertex $v$ of $K$, st $^{0}(v, K)$ - the open star of $v$ in $K$ - is a contractible open set, $\operatorname{st}^{0}(v, K) \times H$ will be homeomorphic to $H$ by [9], so all that is needed is to show that st $^{0}(v, K) \times H_{f}$ is $\mathscr{C}\left(\mathrm{st}^{0}(v, K) \times H\right)$-absorptive. Therefore, let $X$ be a finite-dimensional compactum of $\operatorname{st}^{0}(v, K) \times H$, let $\mathscr{Q}$ be an open cover of $X$ in $\operatorname{st}^{0}(v, K) \times H$ and let $X^{\prime}$ be a closed subset of $X \cap\left(\operatorname{st}^{0}(v, K) \times H_{f}\right)$. Lemma 4 together with the fact that $\mathrm{st}^{0}(v, K) \times H$ is homeomorphic to $H$ establishes that it is sufficient to find an embedding of $X$ in $\operatorname{st}^{0}(v, K) \times H_{f}$ which is limited by $\mathscr{C}$, and is the identity on $X^{\prime}$. Let $\lambda$ be a Lebesgue number for $\mathscr{Q}$ with respect to $X$, and let $p_{H}$ denote the projection of $K \times H$ onto $H$. As noted in the proof of Theorem 4, there exists a sequence $\left\{e_{i}\right\}_{i \in N}$ in $E$ and another sequence $\{n(i)\}_{i \in N}$ in $N$ such that $n(i)-n(i-1) \geqq 2 \operatorname{dim}(X)+2$ for each $i$ and $\left\|\sum_{i=1}^{m} p_{i} \circ p_{H}(x)-p_{H}(x)\right\|<2^{-m-2} \lambda$ for each $m \in N$ and $x \in X$, the rest of the notation being as in the proof of Theorem 4 . Constructing $f_{0}: X \rightarrow H_{f}$ by the same method as used in Theorem 4, 
except for the substitution of $p_{i} \circ p_{H}$ for $p_{i}$, and setting $f=\left(p_{K}, f_{0}\right)$ produces the desired embedding, if $p_{K}$ denotes the projection of $K \times H$ onto $K$.

COROLlary 4. If $K$ is a metric, locally finite-dimensional, simplicial complex such that no vertex-star contains more vertices than $\operatorname{dim}(H)$, then $K \times H_{f}$ is an $H_{f}$-manifold.

Proof. By Theorem 4 of [12], $K \times H$ is an $H$-manifold, so Theorem 5 applies. (This metric is assumed that in the abstract.)

Actually, if a pair $(X, Y)$ of spaces, $Y \subset X$, is called a $\left(H, H_{f}\right)$ manifold pair provided that $X$ is a paracompact $H$-manifold and there is an open cover $\mathscr{C}$ of $X$ by sets $U$ for which there are open embeddings $f_{U}: U \rightarrow H$ such that $f_{U}(U \cup Y)=f_{U}(U) \cap H_{f}$, then the following have been established.

THEOREM 6. The pair $(X, Y)$ is a $\left(H, H_{f}\right)$-manifold pair if and only if $Y$ is a $\mathscr{C}(X)$-complex, $X$ is an $H$-manifold, and the following weak $\mathscr{C}(X)$-absorptivity condition is satisfied: For each finite-dimensional compactum $C$ of $X$, each open cover $\mathscr{U}$ of $C$, and each compact subset $C^{\prime}$ of $C \cap Y$, there is an embedding of $C$ in $Y$ which is limited by $\mathscr{Q}$ and extends the inclusion of $C^{\prime}$. If $(X, Z)$ is another $\left(H, H_{f}\right)$-manifold pair and of is an open cover of $X$, then there is an isotopy of $X$, limited by $\mathscr{Y}$, from the identity to a pair homeomorphism of $(X, Y)$ onto $(X, Z)$.

CoRollary 5. If $(X, Y)$ and $\left(X^{\prime}, Y^{\prime}\right)$ are $\left(H, H_{f}\right)$-manifold pairs, then $\left(X \times X^{\prime}, Y \times Y^{\prime}\right)$ is an $\left(H, H_{f}\right)$-manifold pair.

CoRollary 6. If $(X, Y)$ is an $\left(H, H_{f}\right)$-manifold pair and $K$ is a metric, locally finite-dimensional, simplicial complex such that no vertex-star contains more than $\operatorname{dim}(H)$ vertices, then $(X \times K, Y \times K)$ is an $\left(H, H_{f}\right)$-manifold pair.

\section{REFERENCES}

1. R. D. Anderson, On sigma-compact subsets of infinite-dimensional spaces (to appear, Trans. Amer. Math. Soc.)

2. R. D. Anderson and R. H. Bing, A complete elementary proof that Hilbert space is homeomorphic to the countable infinite product of lines, Bull. Amer. Math. Soc. 74 (1968), 771-792.

3. R. D. Anderson and J. D. McCharen, On extending homeomorphisms to Fréchet manifolds (to appear, Proc. Amer. Math. Soc.)

4. Cz. Bessaga and A. Pelczynski, Some remarks on homeomorphisms of F-spaces, Bull. Polon. Acad. Sci. 10 (1962), 265-270.

5. Cz. Bessaga and A. Pełczynski, Estimated extension theorem, homogeneous collec- 
tions and skeletons, and their applications to topological classification of linear metric spaces and convex sets (to appear, Fund. Math.)

6. T. A. Chapman, Four classes of separable, metric, infinite-dimensional manifolds, Bull. Amer. Math. Soc. 76 (1970), 399-403.

7. - Dense sigma compact subsets of infinite-dimensional manifolds, Trans. Amer. Math. Soc. (to appear).

8. D. W. Henderson, Stable classification of infinite-dimensional manifolds by homotopy type (to appear. Ann. of Math.)

9. D. W. Henderson and R. M. Schori, Topological classification of infinite-dimensional manifolds by homotopy type, Bull. Amer. Math. Soc. 76 (1970), 121-124.

10. D. W. Henderson and J. E. West Triangulated infinite-dimensional manifolds, Bull. Amer. Math. Soc. 76 (1970), 655-660.

11. E. A. Michael, Convex structures and continuous selections, Canad. J. Math. 11 (1959), 556-576.

12. J. E. West, Products of complexes and Fréchet spaces which are manifolds.

Received December 10, 1969. The author was entirely supported by NSF Grant GP-9397 while this research was done.

CORNELL UNIVERSITY

ITHACA, NEW YORK 



\section{PACIFIC JOURNAL OF MATHEMATICS}

EDITORS

H. SAMELSON

Stanford University

Stanford, California 94305

RichaRd PIERCE

University of Washington

Seattle, Washington 98105
J. DUGUNDJI

Department of Mathematics

University of Southern California

Los Angeles, California 90007

RICHARD ARENS

University of California

Los Angeles, California 90024

\section{ASSOCIATE EDITORS}

E. F. BECKENBACH

B. H. NeumanN

F. WOLF

K. YOSHIDA

\section{SUPPORTING INSTITUTIONS}

UNIVERSITY OF BRITISH COLUMBIA

CALIFORNIA INSTITUTE OF TECHNOLOGY

UNIVERSITY OF CALIFORNIA

MONTANA STATE UNIVERSITY

UNIVERSITY OF NEVADA

NEW MEXICO STATE UNIVERSITY

OREGON STATE UNIVERSITY

UNIVERSITY OF OREGON

OSAKA UNIVERSITY

UNIVERSITY OF SOUTHERN CALIFORNIA
STANFORD UNIVERSITY

UNIVERSITY OF TOKYO

UNIVERSITY OF UTAH

WASHINGTON STATE UNIVERSITY

UNIVERSITY OF WASHINGTON

AMERICAN MATHEMATICAL SOCIETY CHEVRON RESEARCH CORPORATION TRW SYSTEMS

NAVAL WEAPONS CENTER 


\section{Pacific Journal of Mathematics}

May, 1970

Johan Aarnes, Edward George Effros and Ole A. Nielsen, Locally compact

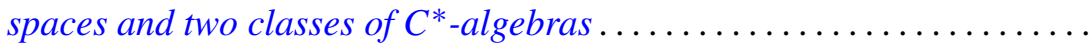

Allan C. Cochran, R. Keown and C. R. Williams, On a class of topological

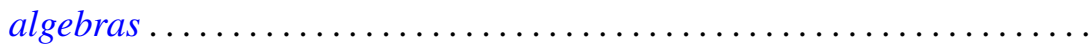

John Dauns, Integral domains that are not embeddable in division rings ....

Robert Jay Daverman, On the number of nonpiercing points in certain

crumpled cubes.....................................

Bryce L. Elkins, Characterization of separable ideals ................

Zbigniew Fiedorowicz, A comparison of two naturally arising uniformities

on a class of pseudo-PM spaces ...........................

Henry Charles Finlayson, Approximation of Wiener integrals of functionals

continuous in the uniform topology ........................

Theodore William Gamelin, Localization of the corona problem ...........

Alfred Gray and Paul Stephen Green, Sphere transitive structures and the

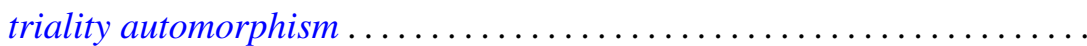

Charles Lemuel Hagopian, On generalized forms of aposyndesis ..........

J. Jakubík, On subgroups of a pseudo lattice ordered group ...............

Cornelius W. Onneweer, On uniform convergence for Walsh-Fourier

series..................................

Stanley Joel Osher, On certain Toeplitz operators in two variables ...

Washek (Vaclav) Frantisek Pfeffer and John Benson Wilbur, On the

measurability of Perron integrable functions............

Frank J. Polansky, On the conformal mapping of variable regions...

Kouei Sekigawa and Shûkichi Tanno, Sufficient conditions for a Riemannian manifold to be locally symmetric ...................

James Wilson Stepp, Locally compact Clifford semigroups ....

Ernest Lester Stitzinger, Frattini subalgebras of a class of solvable Lie

algebras ................................

George Szeto, The group character and split group algebras...

Mark Lawrence Teply, Homological dimension and splitting torsion

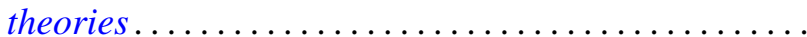

David Bertram Wales, Finite linear groups of degree seven. II ...

Robert Breckenridge Warfield, Jr., An isomorphic refinement theorem for

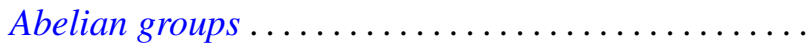

James Edward West, The ambient homeomorphy of an incomplete subspace

of infinite-dimensional Hilbert spaces................

Peter Wilker, Adjoint product and hom functors in general topology ...

Daniel Eliot Wulbert, A note on the characterization of conditional 